\title{
Saturation Throughput Gain in Fixed Multiplexing Radio Networks with Cooperative Retransmission Protocols
}

\author{
Isabella Cerutti, Andrea Fumagalli, and George Ho \\ The OpNeAR Laboratory \\ Erik Jonsson School of Engineering and Computer Science \\ The University of Texas at Dallas \\ E-mails: isabella_cerutti@ieee.org, \{andreaf, georgeho\}@utdallas.edu
}

\begin{abstract}
This paper demonstrates that cooperative ARQ protocols have the desirable property of increasing the saturation throughput of fixed multiplexed access radio networks, subject to non-uniform offered traffic. The throughput gain is found to be increasing as the sourceto-destination channel quality deteriorates and the sourceto-relay channel quality ameliorates. Asymptotically, such gain approaches that of the optimum solution, i.e., the perfect statistical multiplexing.
\end{abstract}

\section{INTRODUCTION}

Many are the known advantages of cooperative communication in radio networking, in which the broadcast radio medium allows for third party nodes to actively help deliver data to the intended destination. One example is the ability to improve the radio channel capacity thanks to the spatial diversity provided by the cooperating nodes, i.e., the relays [1], [2], [3]. Another advantage is the reduced latency of the automatic retransmission request (ARQ) protocols that make use of neighboring relays [4], [5], [6].

In this paper, cooperative communication is investigated in the context of radio networks with fixed multiplexed access. In fixed multiplexing solutions, every source is assigned a dedicated portion of the radio channel bandwidth via time, frequency, or code diversity. By using cooperative communication, relays can dedicate part of their bandwidth to retransmit frames on behalf of the actual source. To quantify this phenomenon, the saturation throughput of a fixed multiplexing radio network that makes use of a cooperative ARQ protocol is estimated analytically using a flow model.

The analytical model demonstrates that cooperative communication has the desirable property of alleviating

This research is supported in part by NSF Grants No. ECS0225528, CNS-0435429, and the Italian Ministry of University (MIUR) under FIRB project No. RBNE01KNFP. one of the main drawbacks of fixed multiplexing, i.e., the lack of flexible bandwidth allocation when supporting non-uniform and variable offered traffic. Moreover, it helps identify the conditions under which cooperative communication yields asymptotically optimal bandwidth allocation (i.e., that of the perfect statistical multiplexing). These conditions include a degraded signal quality on the source-destination channel and a good signal quality on the channel between the cooperating nodes.

\section{The Cooperative ARQ Protocol in FiXed Time Division MultipleXed ACCEss Radio NETWORK}

Define the access radio network as consisting of $n$ nodes, i.e., sources $S_{0}, S_{1}, \ldots, S_{n-1}$. They all send data frames to the same destination, $D$. One single broadcast radio channel is available and shared by all the sources. Propagation time over the channel is considered to be negligible. Over the channel, time is divided into slots of equal size. During one slot, exactly one data frame may be transmitted by one source, and one positive acknowledgment may be transmitted by $D$. Each node is assigned one slot for transmission every $n$ slots, i.e., access control is based on fixed Time Division Multiplexing (TDM). Since data frames may be corrupted and not received correctly by $D$ during their first transmission, they are (re)transmitted until they are successfully received by $D$ using a simple stop and wait ARQ protocol. Note that if sources behave in a non-cooperative way, multiple transmissions of the same data frame are performed by the source itself in its own slots.

The following cooperative ARQ protocol is considered. Each source is in the reception mode at all times, except when transmitting during its own assigned slots. When generated, the original data frame is first transmitted by its own source. Copies of the transmitted 
original data frame ${ }^{1}$ are created and temporarily stored at the other sources that have successfully overheard the ongoing transmission. Copies of the overheard data frames are discarded when older than $n-1$ slots. Positive acknowledgments from $D$ are broadcast to all sources. When it is one source's turn to transmit, two cases are possible: the source either has or does not have its own data frame awaiting transmission in the transmitter buffer. In the former case, its oldest data frame is transmitted. In the latter case, the oldest outstanding data frame $^{2}$ copy that was overheard during the last $n-1$ slots is retransmitted. In the latter case, the source is acting as a relay for some other source. Note that multiple relays may cooperate to help the same source, i.e., copies of the same data frame may be retransmitted by distinct relays, before the source can attempt transmission again $n$ slots later.

\begin{tabular}{|c|c|c|c|c|c|c|c|c|}
\hline $\mathrm{D}_{0}$ & $\mathrm{D}_{1}$ & $\mathrm{ACK}$ & $\mathrm{C}_{0,2}$ & & & $\mathrm{C}_{0,4}$ & $\mathrm{ACK}$ & $\mathrm{D}_{0}^{\prime}$ \\
\hline $\mathrm{S}_{0}$ & $\mathrm{~S}_{1}$ & $\mathrm{~S}_{2}$ & $\mathrm{~S}_{3}$ & $\mathrm{~S}_{4}$ & $\mathrm{~S}_{0}$ & time \\
\hline
\end{tabular}

Fig. 1. Fixed time division multiplexing with cooperation

Fig. 1 illustrates the case when sources $S_{2}$ and $S_{4}$ transmit copies $C_{0,2}$ and $C_{0,4}$, respectively, of the same original data frame $D_{0}$ sent by $S_{0}$. In the example, source $S_{1}$ transmits its own data frame $D_{1}$ and does not cooperate. Neither source $S_{3}$ cooperates, because it did not receive data frame $D_{0}$ correctly from $S_{0}$ [7].

Note that the presented cooperative protocol is just one of many possible variants. For example, it is possible to arbitrarily limit the number of relays that may help a given source, in order to reduce the number of data frame copies generated and the amount of time devoted to performing relay work. Also, it is possible to add redundancy to the frames through coding, in order to increase the reliability of the transmissions and thus reduce the latency [7].

It is interesting to observe that cooperation may be used in this scenario to achieve flexible bandwidth allocation. For example, imagine that temporarily one source is generating more traffic than average, and the other sources are generating less traffic than average. Retransmissions of unsuccessful frames will be performed primarily by the latter group of sources. By having the other sources retransmitting its own unsuccessful frames, the former source - the one generating aboveaverage traffic - will experience a less severe bandwidth constraint originating from the fixed bandwidth multi-

\footnotetext{
${ }^{1}$ Note that copies of transmitted copies are not allowed.

${ }^{2} \mathrm{~A}$ data frame is defi ned as outstanding after being transmitted by its own source and before being correctly received by $D$.
}

plexing. While this advantage is intuitive, it is important to quantify the throughput gain that the presented cooperative ARQ protocol may yield under non-uniform traffic condition. This aspect is addressed in the next section.

\section{Throughput Derivation}

This section presents a model for assessing the saturation throughput under non-uniform traffic that may be achieved in the fixed TDM access network with the cooperative ARQ protocol described in Section II. For simplicity of derivation, the model assumes that all acknowledgment frames are received correctly by the sources. It is possible to extend the presented model to account for lost acknowledgments. However, given the complexity of such derivation and its modest qualitative impact on the results, this case is not considered here.

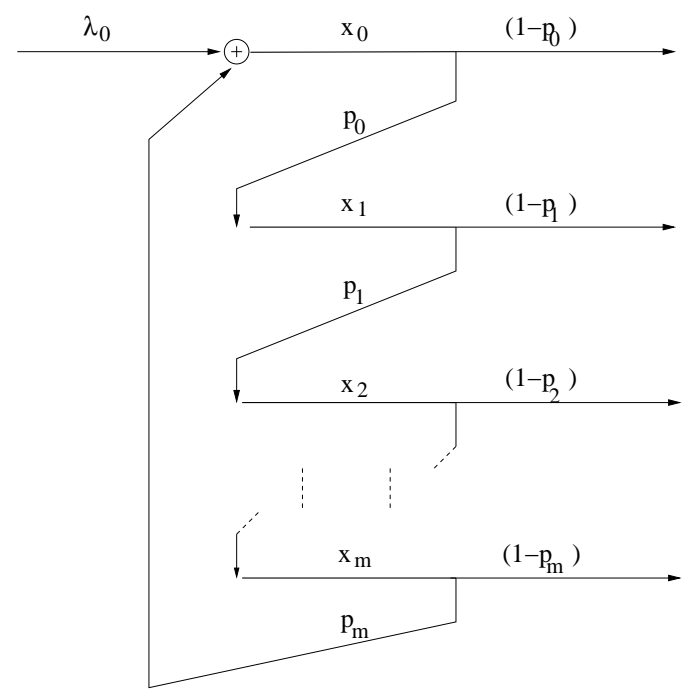

Fig. 2. Flow of frames: ideal source-relay channel

Assume that all frame error probabilities due to transmission in the network are time-invariant. Define the frame error probability from $S_{i}$ to $D$ as $p_{i}, i=0, \ldots, n-$ 1. Define the frame error probability from $S_{0}$ to $S_{i}$ as $q_{i}, i=1, \ldots, n-1$.

Note that the most adverse condition from the saturation throughput point of view is experienced when only one source is active, and the remaining $m=n-1$ are not generating traffic. For this reason, it is assumed that the only active source is $S_{0}$, with a frame generation rate of $\lambda_{0}$. The other $m$ nodes act as potential relays for $S_{0}$.

Figs. 2 and 3 show the flow of frames (original data and copies) assuming an ideal (i.e., $q_{i}=0, \forall i$ ) and nonideal (i.e., $q_{i} \neq 0, i \in\{1, \ldots, n\}$ ) source-relay channel, respectively. The saturation throughput for the two cases is derived next. 


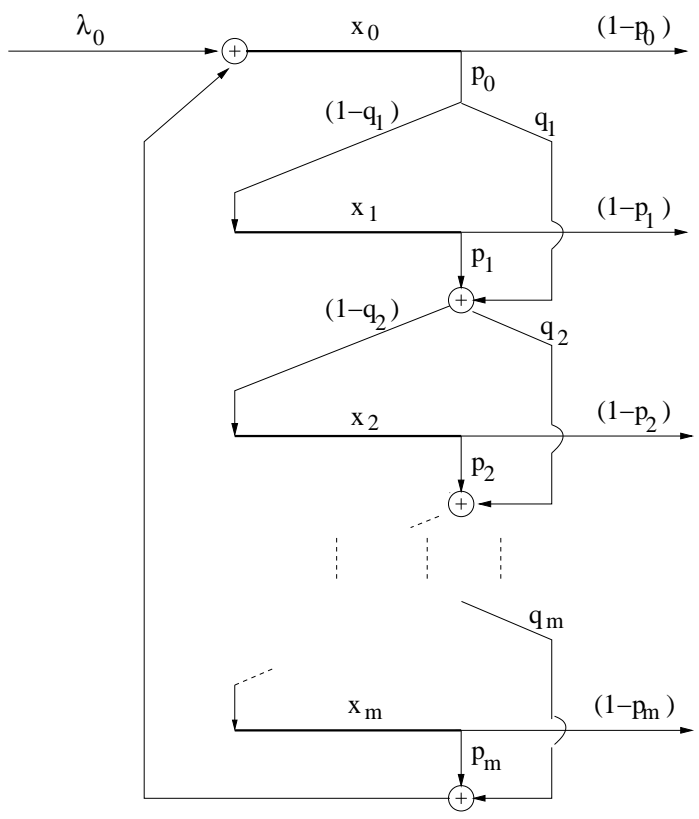

Fig. 3. Flow of frames: non-ideal source-relay channel

\section{A. Ideal Source-Relay Channel}

From Fig. 2, the rate of transmitted frames at $S_{0}$ is

$$
x_{0}=\lambda_{0}+p_{m} \cdot x_{m},
$$

where $x_{m}$ is the rate of transmitted frames at relay $m$, i.e.,

$$
x_{m}=p_{m-1} \cdot x_{m-1} .
$$

The rate of transmitted frames at relay $m-1$ is

$$
x_{m-1}=p_{m-2} \cdot x_{m-2} .
$$

By recursion, the rate at relay $i \geq 1$ is

$$
x_{i}=p_{i-1} \cdot x_{i-1}=\prod_{j=1}^{i} p_{j-1} \cdot x_{0} .
$$

Substituting (4) in (1) and solving for $x_{0}$

$$
\begin{gathered}
x_{0}=\lambda_{0}+p_{m} \prod_{j=1}^{m} p_{j-1} \cdot x_{0} \\
x_{0}=\frac{\lambda_{0}}{1-p_{m} \prod_{j=1}^{m} p_{j-1}} .
\end{gathered}
$$

By the nature of fixed multiplexing, each node is reserved one $n$-th of the channel bandwidth, i.e.,

$$
x_{i} \leq \frac{1}{n} \forall i
$$

Noting that $x_{0} \geq x_{i}, \forall i$, condition (7) may be used to compute the saturation throughput at $S_{0}$

$$
\lambda_{\max }^{c}=\frac{1-\prod_{j=0}^{m} p_{j}}{n} .
$$

For the symmetric channel case, i.e., $p_{i}=p \forall i$, the saturation throughput is

$$
\lambda_{\max }^{c}=\frac{1-p^{m+1}}{n}=\frac{(1-p) \sum_{j=0}^{m} p^{j}}{n} .
$$

The result in (9) shows that fixed TDM without cooperation $(m=0)$ yields a saturation throughput of

$$
\lambda_{\max }^{f}=\frac{(1-p)}{n} .
$$

Overall, for the symmetric channel case the saturation throughput gain of cooperative over non-cooperative ARQ protocol in fixed TDM is

$$
\gamma^{c f}=\sum_{j=1}^{m} p^{j}
$$

\section{B. Non-Ideal Source-Relay Channel}

Fig. 3 shows the flows of frames when $q_{i} \neq 0 \forall i$. From Fig. 3,

$$
x_{1}=p_{0}\left(1-q_{1}\right) x_{0},
$$

$$
\begin{aligned}
x_{2} & =\left(1-q_{2}\right)\left(p_{1} \cdot x_{1}+p_{0} \cdot q_{1} \cdot x_{0}\right)= \\
& =\frac{\left(1-q_{2}\right)}{\left(1-q_{1}\right)}\left[p_{1}\left(1-q_{1}\right)+q_{1}\right] x_{1},
\end{aligned}
$$

$$
\begin{aligned}
x_{3} & =\left(1-q_{3}\right)\left(p_{2} \cdot x_{2}+p_{1} \cdot q_{2} \cdot x_{1}+p_{0} \cdot q_{2} \cdot q_{1} \cdot x_{0}\right)= \\
& =\frac{\left(1-q_{3}\right)}{\left(1-q_{2}\right)}\left[p_{2}\left(1-q_{2}\right)+q_{2}\right] x_{2} .
\end{aligned}
$$

By recursion, at relay $i \geq 1$

$$
\begin{aligned}
x_{i} & \left.=x_{i-1} \frac{\left(1-q_{i}\right)}{\left(1-q_{i-1}\right)}\left[p_{i-1}\left(1-q_{i-1}\right)+q_{i-1}\right]\right)= \\
& =x_{0} p_{0}\left(1-q_{i}\right) \prod_{j=1}^{i-1}\left[p_{j}\left(1-q_{j}\right)+q_{j}\right]
\end{aligned}
$$

Using the recursive result in (15), the rate of frames transmitted at $S_{0}$ is

$$
x_{0}=\lambda_{0}+x_{m} \frac{p_{m}\left(1-q_{m}\right)+q_{m}}{1-q_{m}} .
$$

By replacing $x_{m}$ with (15) for $i=m$, and solving for $x_{0}$

$$
x_{0}=\frac{\lambda_{0}}{1-p_{0} \prod_{j=1}^{m}\left[p_{j}\left(1-q_{j}\right)+q_{j}\right]} .
$$

By imposing constraint (7)

$$
\lambda_{\max }^{c}=\frac{1-p_{0} \prod_{j=1}^{m}\left[p_{j}\left(1-q_{j}\right)+q_{j}\right]}{n} .
$$

Note that (18) when $q=0$ becomes (8). 
For the symmetric channel case, i.e., $p_{i}=p \forall i$ and $q_{i}=q \forall i$, the saturation throughput is

$$
\lambda_{\max }^{c}=\frac{1-p[p(1-q)+q]^{m}}{n} .
$$

Overall, for the symmetric channel case the saturation throughput gain of cooperative over non-cooperative ARQ protocol in fixed TDM is

$$
\gamma^{c f}=\sum_{j=1}^{m}[\sqrt[m]{p}(p(1-q)+q)]^{j} .
$$

\section{Perfect Statistical Multiplexing BENCHMARK}

For benchmarking the performance of the fixed TDM access, the perfect statistical TDM case is considered. This is the case when slots are dynamically assigned to sources to perfectly match their offered traffic. This solution may not be practical in reality, but it represents an upper bound on saturation throughput.

Note that cooperative ARQ protocols may be applied to the statistical TDM case too. However - as shown in the following derivations - cooperation does not yield any gain on saturation throughput when the channel quality is symmetric. The saturation throughput is derived for both the ideal and non-ideal source-relay channel cases.

\section{A. Ideal Source-Relay Channel}

Under perfect statistical multiplexing all sources dynamically share the whole channel bandwidth, i.e.,

$$
\sum_{j=0}^{m} x_{j} \leq 1
$$

Using (4) and (6), the bandwidth constraint is

$$
\frac{\lambda_{0}\left(1+\sum_{j=1}^{m} \prod_{i=0}^{j-1} p_{i}\right)}{1-\prod_{j=0}^{m} p_{j}} \leq 1,
$$

and the saturation throughput is

$$
\lambda_{\max }^{s}=\frac{1-\prod_{j=0}^{m} p_{j}}{1+\sum_{j=1}^{m} \prod_{i=0}^{j-1} p_{i}} .
$$

In the absence of cooperation, (23) reduces to

$$
\lambda_{\max }^{s}=1-p_{0} .
$$

For the symmetric channel case, i.e., $p_{i}=p \forall i$, it is possible to verify that (23) reduces to

$$
\lambda_{\max }^{s}=1-p .
$$

This result demonstrates that cooperative ARQ protocols cannot yield any throughput gain in the case of statistical TDM with symmetric channel.
Overall, for the symmetric channel case the saturation throughput loss of fixed TDM with the cooperative ARQ protocol with respect to perfect statistical TDM is

$$
\gamma^{c s}=\frac{\sum_{j=0}^{m} p^{j}}{n}-1
$$

\section{B. Non-Ideal Source-Relay Channel}

Using (15) and (17), constraint (21) becomes

$$
\frac{\lambda_{0}\left(1+p_{0} \sum_{j=1}^{m}\left(1-q_{j}\right) \prod_{i=1}^{j-1}\left(p_{i}\left(1-q_{i}\right)+q_{i}\right)\right)}{1-p_{0} \prod_{j=1}^{m}\left(p_{j}\left(1-q_{j}\right)+q_{j}\right)} \leq 1,
$$

and the saturation throughput is

$$
\lambda_{\max }^{s}=\frac{1-p_{0} \prod_{j=1}^{m}\left(p_{j}\left(1-q_{j}\right)+q_{j}\right)}{1+p_{0} \sum_{j=1}^{m}\left(1-q_{j}\right) \prod_{i=1}^{j-1}\left(p_{i}\left(1-q_{i}\right)+q_{i}\right)} .
$$

In the absence of cooperation, (28) reduces to

$$
\lambda_{\max }^{s}=1-p_{0} .
$$

For the symmetric channel case, i.e., $p_{i}=p \forall i$ and $q_{i}=q \forall i$, (28) reduces to

$$
\lambda_{\max }^{s}=1-p .
$$

This result demonstrates that cooperative ARQ protocols cannot yield any throughput gain in the case of statistical TDM with symmetric and non-ideal source-relay channel.

For the symmetric channel case, the saturation throughput loss of fixed TDM with the cooperative ARQ protocol with respect to perfect statistical TDM is the same of (26).

\section{Some Numerical Examples}

Some numerical examples are presented in this section to help visualize the equations obtained so far. Results are shown for the ARQ Protocol in fixed TDM access radio network with and without cooperation, and in a perfect statistical TDM.

The results are presented for the symmetric channel case only, i.e., $p_{i}=p \forall i$ and $q_{i}=q \forall i$.

Figs. 4 and 5 indicate the regions of stability when using statistical TDM, fixed TDM with and without cooperation. Stability is ensured when the offer load does not exceed the saturation throughput. Two nodes only $(n=2)$ are considered: $S_{0}$ with frame arrival rate $\lambda_{0}$ and $S_{1}$ with frame arrival rate $\lambda_{1}$. Each source acts as a relay for the other. In the figures, the frame transmission error probabilities are $p=0.3$ and $p=0.8$, respectively. The case of ideal and non-ideal relay-source $(q=0.8)$ 


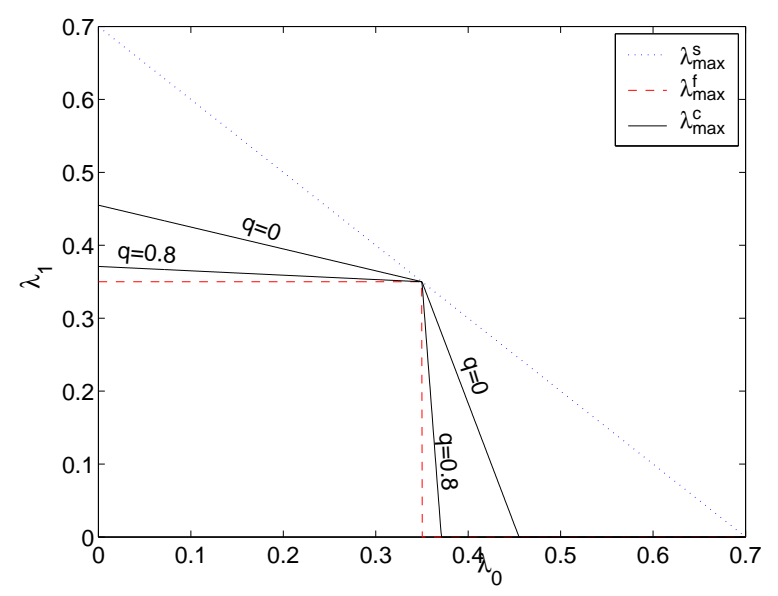

Fig. 4. $\lambda_{\max }^{s}, \lambda_{\max }^{f}$, and $\lambda_{\max }^{c}$ vs. $\lambda_{0}$ and $\lambda_{1}$ when $n=2, p=0.3$

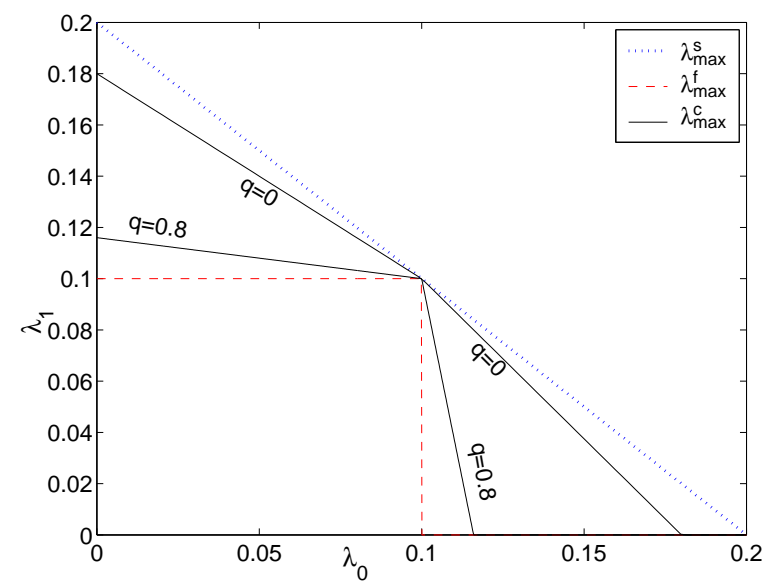

Fig. 5. $\lambda_{\max }^{s}, \lambda_{\max }^{f}$, and $\lambda_{\max }^{c}$ vs. $\lambda_{0}$ and $\lambda_{1}$ when $n=2, p=0.8$

channel is considered. For the perfect statistical TDM, the stability region, i.e., the region in which both arrival rates do not overcome their saturation throughput, is a triangle. For fixed TDM without cooperation, it is a square. For fixed TDM with cooperation, the stability region is intermediate with respect to the previous two. The size of this intermediate region is highly sensitive to the values of $p$ and $q$.

For the favorable network scenario of $p=0.8$ and $q=0$, Fig. 6 (Fig. 7) plots the saturation throughput gain (loss) of fixed TDM with cooperation when compared to fixed (statistical) TDM without cooperation. Significant gains are found when the offered traffic is not uniform, i.e., the throughput gain may be as high as $80 \%$ when compared to the non-cooperative fixed TDM case. The loss may be as high as $10 \%$ when compared to perfect statistical TDM.

Fig. 8 plots the saturation throughput of $S_{0}$ versus $p$ when $\lambda_{i}=0, \forall i>0$ and the source-relay channel is ideal, i.e., $q=0$. All three multiplexing solutions are shown considering $n=2,5,10$. Note the saturation

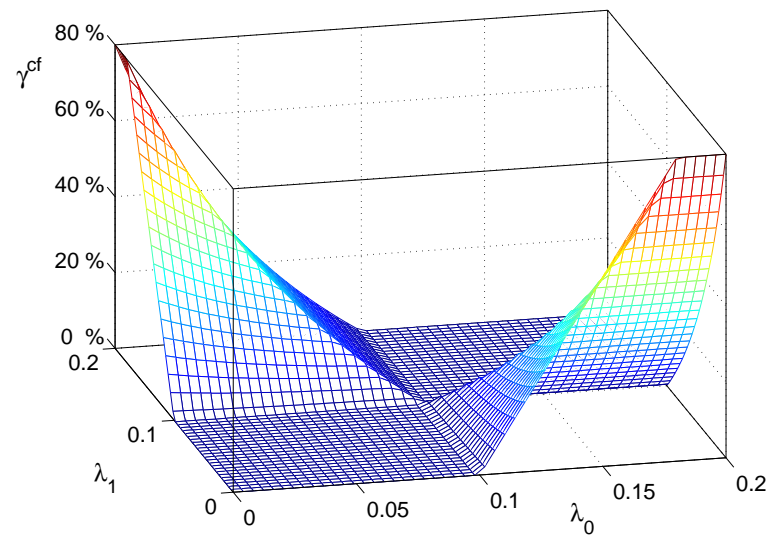

Fig. 6. $\gamma^{c f}$ vs. $\lambda_{0}$ and $\lambda_{1}$ when $n=2, p=0.8, q=0$

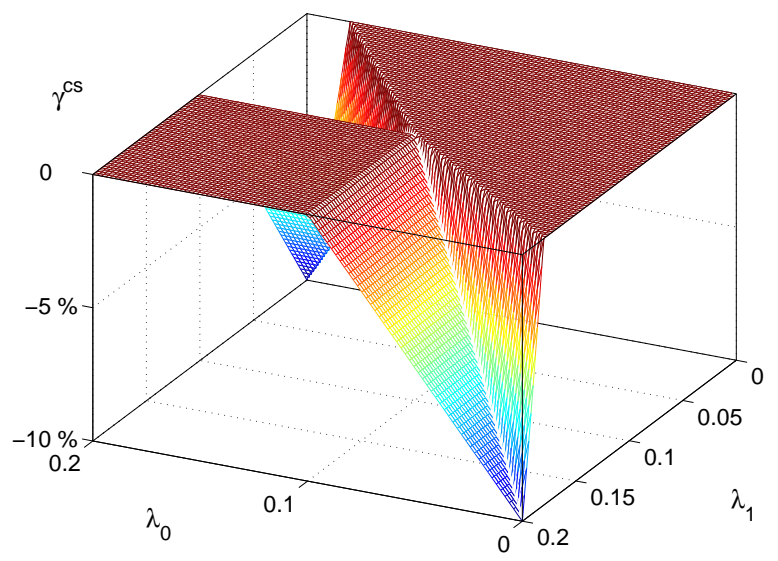

Fig. 7. $\gamma^{c s}$ vs. $\lambda_{0}$ and $\lambda_{1}$ when $n=2, p=0.8, q=0$

throughput of fixed TDM that is inversely proportional to $p$. The plots confirm that cooperation helps increase the saturation throughput of fixed multiplexing, especially in the presence of severely degraded source-destination channel quality $(p \rightarrow 1)$.

Fig. 9 plots the saturation throughput using the same assumptions of Fig. 8, but now assuming $q \neq 0$ and $p=0.8$. The plots document that the advantage of using the cooperative ARQ protocol in fixed TDM is highly dependent on the source-relay channel quality $q$.

Figs. 10 and 11 plot the first derivative of the saturation throughput (in (19) and (30)) with respect to $p$ in the case of non-ideal source-relay channel, when $n=2,5$, respectively. The plots show that when $q \rightarrow 0$ and $p \rightarrow 1$, the saturation throughput first derivative for the cooperative ARQ protocol approaches that of the perfect statistical multiplexing case.

\section{CONCLUSION}

The paper demonstrated that the use of cooperative ARQ protocols in access radio networks may mitigate 


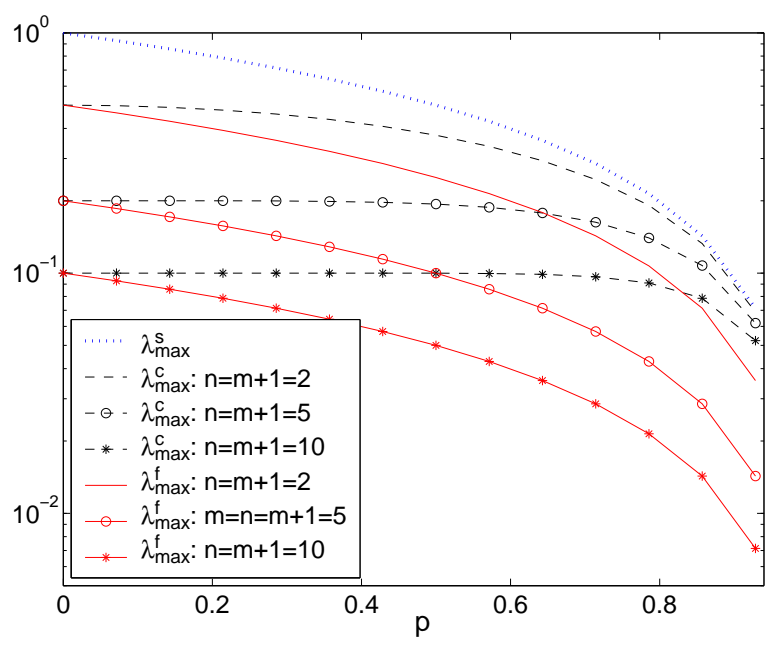

Fig. 8. $\quad \lambda_{\max }^{c}, \lambda_{\max }^{f}$, and $\lambda_{\max }^{s}$ vs. $p$ when $q=0$

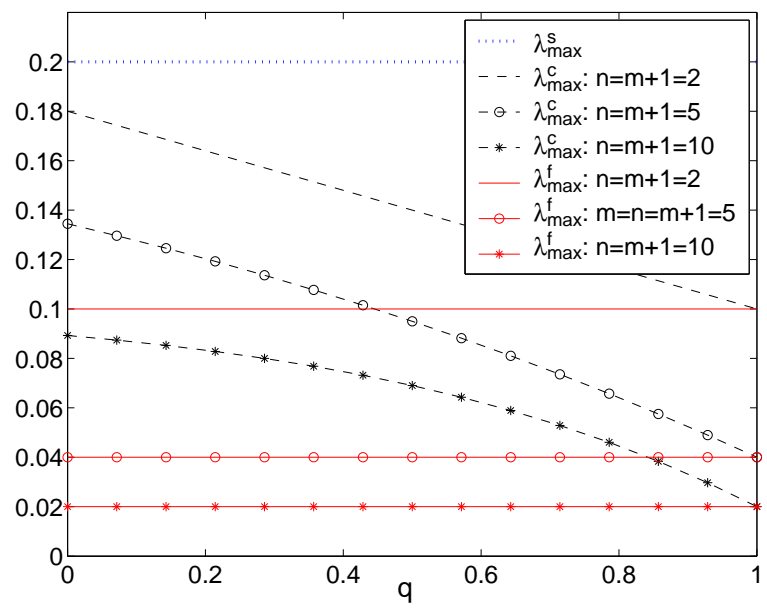

Fig. 9. $\quad \lambda_{\max }^{c}, \lambda_{\max }^{f}$, and $\lambda_{\max }^{s}$ vs. $q$ when $p=0.8$

the well-known drawback of fixed bandwidth multiplexing, i.e., the lack of flexible bandwidth allocation when traffic is not uniform. It was demonstrated that as the quality of the channel from source to destination worsens and the quality of the channel from source to relay improves, the use of a cooperative ARQ protocol may improve the saturation throughput of fixed multiplexing, asymptotically reaching the best performance possible, i.e., that of the perfect statistical multiplexing.

This result is consistent with numerous other publications that have demonstrated the benefits of cooperative communications in channels with poor signal-to-noise ratio [2], [6], [8], [9], [10].

\section{REFERENCES}

[1] A. Sendonaris, E. Erkip, and B. Aazhang, "User cooperation diversity-Part I: System description," IEEE Trans. on Comm., pp. 1927-1938, November 2003.

[2] J. N. Laneman, G. W. Wornell, and D. N. C. Tse, "An effi cient protocol for realizing cooperative diversity in wireless networks," in Proc. of IEEE ISIT, 2001, p. 294.

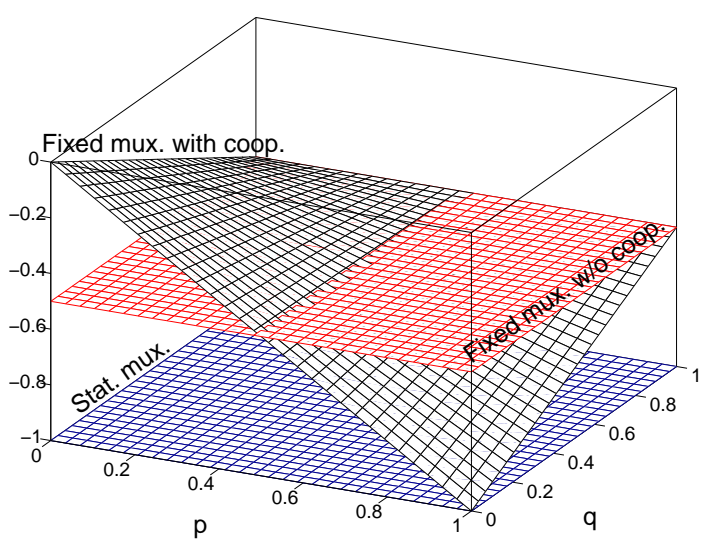

Fig. 10. $\partial \lambda_{\max }^{c} / \partial p, \partial \lambda_{\max }^{f} / \partial p$, and $\partial \lambda_{\max }^{s} / \partial p$ vs. $p$ and $q$, when $n=m+1=2$

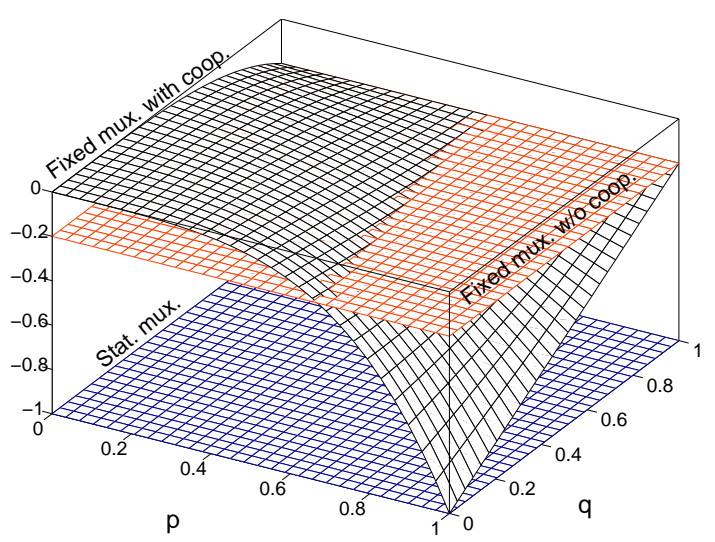

Fig. 11. $\partial \lambda_{\max }^{c} / \partial p, \partial \lambda_{\max }^{f} / \partial p$, and $\partial \lambda_{\max }^{s} / \partial p$ vs. $p$ and $q$ when $n=m+1=5$

[3] M. Janani, A. Hedayat, T. E. Hunter, and A. Nosratinia, "Coded cooperation in wireless communications: space-time transmission and iterative decoding," IEEE Transactions on Signal Processing, vol. 52, no. 2, pp. 362 - 371, Feb. 2004.

[4] E. Zimmermann, P. Herhold, and G. Fettweis, "The impact of cooperation on diversity-exploiting protocols," in Proc. of 59th IEEE Vehicular Technology Conference (VTC Spring), 2004.

[5] B. Zhao and M. C. Valenti, "Practical relay networks: a generalization of hybrid-ARQ," IEEE Journal on Selected Areas in Communications, vol. 23, no. 1, pp. 7 - 18, Jan. 2005.

[6] P. Gupta, I. Cerutti, and A. Fumagalli, "Three transmission scheduling policies for a cooperative ARQ protocol in radio networks," in Proc. WNCG conference, October 2004.

[7] I. Cerutti, P. Gupta, and A. Fumagalli, "Cooperative ARQ protocols in slotted radio networks," The University of Texas at Dallas, Tech. Rep. UTD/EE-12/2005, 2005.

[8] E. Zimmermann, P. Herhold, and G. Fettweis, "On the performance of cooperative relaying protocols in wireless networks," Eur. Trans. on Telecom. (ETT), vol. 16, no. 1, pp. 17-35, 2005.

[9] A. Nosratinia, T. Hunter, and A. Hedayat, "Cooperative communication in wireless networks," IEEE Comm. Magazine, vol. 42, no. 10 , pp. $74-80,2004$.

[10] M. Tacca, P. Monti, and A. Fumagalli, "Cooperative and noncooperative ARQ protocols for microwave recharged sensor nodes," in Proc. EWSN, 2005. 